\title{
An uncommon obliterative lung disease: Swyer-James-MacLeod syndrome
}

\author{
Katherine M. Wojcicki ${ }^{1}$ - Ariel D. Sindel ${ }^{1}$ - Andrew C. Berry ${ }^{2} \cdot$ Robert H. Krieger $^{1}$ • \\ Warren L. Reuther III $^{3}$
}

Received: 7 April 2015/Accepted: 9 April 2015/Published online: 5 May 2015

(C) SIMI 2015

A 42-year-old woman presented with substernal chest pain, productive cough with yellow sputum, and shortness of breath. Chest pain was present for 1 day, and worsened with deep inhalation and movement. The patient denied fever, chills, and diaphoresis. Physical examination revealed a well-appearing women in no apparent distress. Auscultation of the lungs demonstrated moderate crackles in the right lower lung, and chest examination was positive for mild tenderness to palpation along the right anterior chest wall. The remainder of the physical examination was unremarkable. A complete blood count with differential, complete metabolic panel, cardiac enzymes, and electrocardiogram were unremarkable. Anterior-posterior chest $\mathrm{X}$-ray study demonstrated diminished right thoracic cage volume and diminished right lung volumes compared to the left (Fig. 1a). Computed tomography angiogram (CTA) revealed a hyperlucent right lung with diminished vascularity, and an overall reduction in right lung volume compared to the left (Fig. 1b). Ventilation-perfusion scan demonstrated matched defects. Specifically, there was decreased radioisotope uptake in the right lung on both the ventilation and perfusion images, compatible with a destructive process of the entire right lung (Fig. 1c, d). Clinical appearance and imaging supported a diagnosis of

Katherine M. Wojcicki

Wojcickk@my.canisius.edu

1 Kansas City University of Medicine and Biosciences (KCUMB), 1750 E. Independence Ave., Kansas City, MO 64106, USA

2 Department of Medicine, University of South Alabama, Mobile, AL, USA

3 Department of Radiology, Palms West Hospital, West Palm Beach, FL, USA
Swyer-James-MacLeod syndrome, a rare acquired obliterative lung syndrome.

Swyer-James-MacLeod syndrome (SJMS) is an uncommon obliterative lung disease that is a rare complication of a lower respiratory tract infection during childhood [1]. This syndrome is most often seen in children, but can rarely present in adults with a past medical history of recurrent respiratory infections. SJMS can be caused by numerous microbes including adenovirus types 3,7, and 21, paramyxovirus morbillivirus, Bordetella pertussis, Mycoplasma pneumoniae, Mycobacterium tuberculosis, and influenza A [2, 3]. These infections can cause post-infectious bronchiolitis obliterans, and may also cause hypoplasia or agenesis of the pulmonary vasculature. In the latter scenario, hypoperfusion of the pulmonary parenchyma will result in unilateral hyperlucency of the lung on radiographic images [2,3]. The symptoms of SJMS can vary from asymptomatic to chronic or recurring lung infections, dyspnea, or hemoptysis [1]. Pathogenesis involves inflammation of the respiratory bronchioles, leading to bronchiole wall fibrosis and concurrent narrowing of the bronchiole lumens. Fibrosis in the interalveolar septae is responsible for the destruction of the pulmonary capillary beds, which results in decreased perfusion to the pulmonary artery, leading to pulmonary artery hypoplasia [1].

Diagnosis is made following radiographic evidence (plain film and CT) of the classic SJMS triad: which includes a unilateral hyperlucent lung, diffusely decreased ventilation, and matching decreased perfusion in the affected lung [3]. Unilateral lung hypoplasia, destruction of the pulmonary parenchyma and air trapping (bronchiectasis) are also likely to be visualized on CT imaging [1, 2]. Abnormal pulmonary arterial vasculature characterized by decreased caliber may be visualized if CTA of the pulmonary vasculature is performed [2]. The treatment of SJMS is often conservative, and typically involves 
Fig. 1 Anterior-posterior plain film of chest (a) demonstrating diminished right thoracic cage volume and diminished right lung volume compared to the left. CT of chest with IV contrast (b) illustrating hyperlucency of the right lung with diminished right lung vascularity and volume. There is right upper, middle, and lower lobe bronchiectasis. Pulmonary ventilation study (c) following inhalation of technetium $99 \mathrm{~m}$ DTPA aerosol illustrates increased tracer accumulation in right upper lung, with only moderate accumulation in the right middle lung. The right lower lung shows large fields without appreciable tracer accumulation. The left lung produces a normal response of homogenous tracer accumulation throughout. Pulmonary perfusion study (d) following IV administration of technetium 99 MAA demonstrates heterogeneous distribution of tracer in the right lung which matches that of the ventilation study. There is normal homogenous tracer distribution throughout the left lung
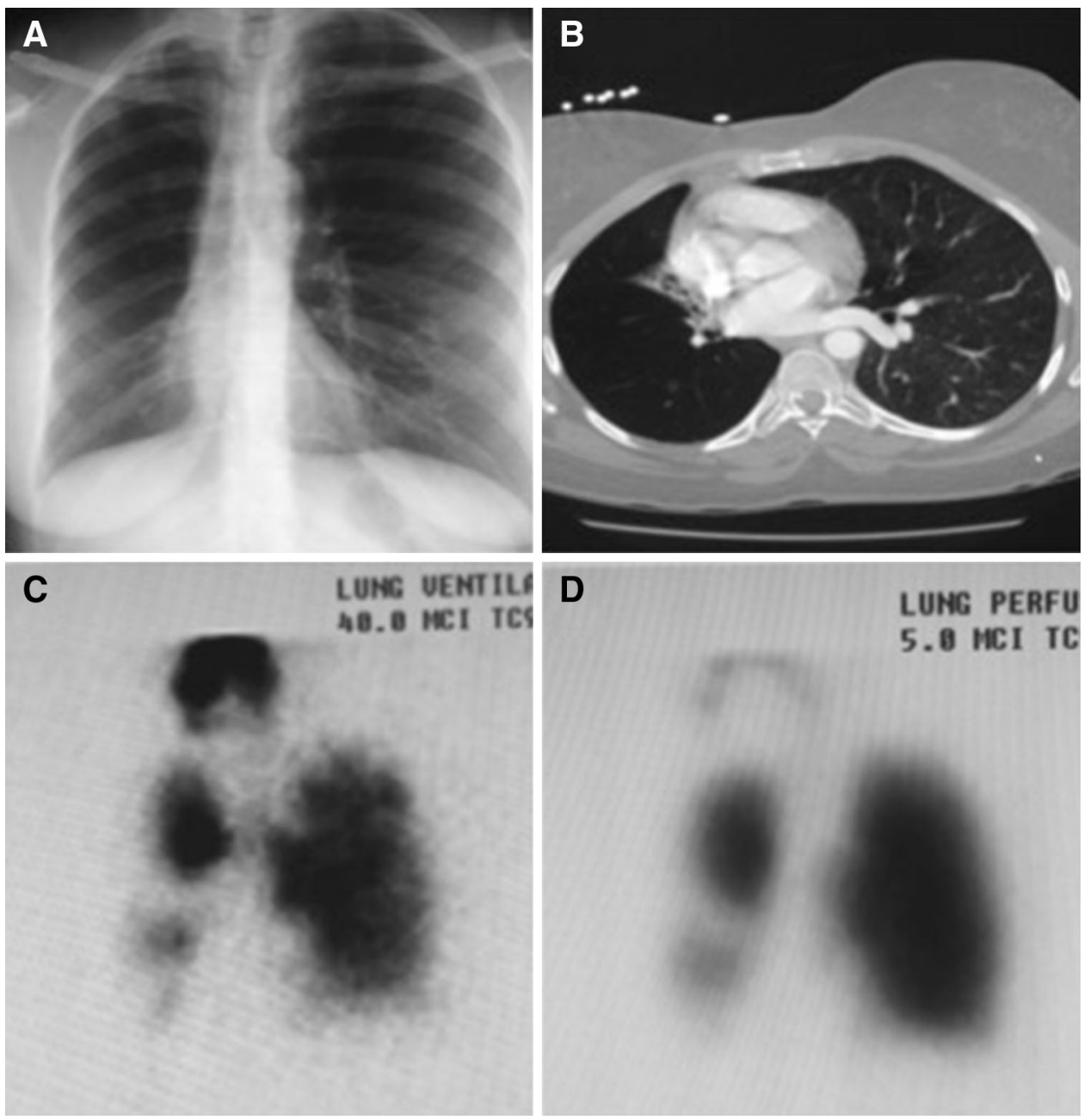

managing current, and preventing future pulmonary infections. The administration of the influenza and pneumococcal vaccines is, therefore, recommended [2]. Surgical consideration such as pneumonectomy, lobectomy, or segmentectomy has been documented to improve lung function and quality of life in patients who suffer from uncontrolled infections or severe lung damage [4].

This case outlines the unique presentation of SJMS in an adult presenting with shortness of breath and unilateral lung hypoplasia on CT imaging. Although commonly believed to be a syndrome diagnosed during childhood, SJMS can be diagnosed during adult life, illustrating the importance of considering SJMS in both children and adults with recurrent respiratory infections and shortness of breath. With an accurate diagnosis, proper preventative measures can be taken to decrease future complications and improve the quality of life in individuals with SJMS.

\section{Conflict of interest None.}

Statement of human and animal rights All procedures performed in human participants were in accordance with the ethical standards of the institutional research committee and with the 1964 Helsinki declaration and its later amendments or comparable ethical standards. This article does not contain any studies with animals performed by any of the authors.

Informed consent Informed consent was obtained from all individual participants included in the study.

\section{References}

1. Hajsadeghi SH, Chitsazan M, Pouraliakbar HP, Kerman SRJ (2010) Swyer-James-MacLeod syndrome presenting with pulmonary hypertension. Iranian Cardiovasc Res J 4(3):134-138

2. Capela C, Gouveia P, Sousa M, Regadas MJ (2011) Adult diagnosis of Swayer-James-MacLeod syndrome: a case report. J Med Case Rep 5(2):1-4

3. Damle NA, Mishra R, Wadhwa JK (2012) Classic imaging triad in a very young child with Swyer-James syndrome. Nuclear Med Mol Imaging 46(2):115-118

4. LucasdeSilva PS, Lopes R, Neo HM (2012) Swyer-JamesMacLeod syndrome in a surgically treated child: a case report and brief literature review. J Pediatr Surg 47(2):e17-e22 